\section{Control of the cell cycle progression by the MAPK Hog1}

\author{
Josep Clotet, 1 Joan Vendrell,2,3 \\ Xavier Escoté2,3
}

1Departament de Ciències Bàsiques, Universitat Internacional de Catalunya, Sant Cugat del Vallès; 2Unitat de Recerca; Hospital Universitari Joan XXIII, IISPV, Tarragona; ${ }^{3}$ CIBERDEM; Instituto de Salud Carlos III, Spain

\section{Abstract}

Eukaryotic cells coordinate various intracellular activities in response to environmental stresses, activating an adaptive program to maximize the probability of survival and proliferation. Cells transduce diverse cellular stimuli by multiple mitogen-activated protein kinase (MAPK) cascades. MAPK are key signal transduction kinases required to respond to stress. A prototypical member of the MAPK family is the yeast high osmolarity glycerol (Hog1). Activation of Hog1 results in the generation of a set of adaptive responses that leads to the modulation of several aspects of cell physiology that are essential for cell survival, such as gene expression, translation, and morphogenesis. This review focuses on the control of cell cycle progression by Hogl which is critical for cell survival in response to stress conditions.

\section{The high osmolarity glycerol pathway}

Cells are constantly exposed to stress situations such as changes in temperature, $\mathrm{pH}$, radiation, availability of nutrients, access to oxygen and changes in osmotic pressure. Cells are able to coordinate intracellular activities in order to respond to such stresses. Mitogenactivated protein kinases (MAPKs) are a conserved protein family that sense and respond to extracellular environmental changes. MAPK Activation leads to the generation of a set of adaptive responses that involves the modulation of several physiological processes such as changes in gene transcription, protein regulation and control of cell cycle progression, which allow cells to adapt to the new environmental conditions.1,2 When cells are subjected to hyperosmotic shock, they lose water in a passive diffusion process and a specific MAPK pathway is activated: the high osmolarity glycerol (HOG) pathway. To fight against the loss of water, cells have developed a battery of mechanisms and the HOG pathway develops a central function in this process. ${ }^{3-5}$

Activation of the HOG pathway elicits a platform for cell osmoadaptation, which consists of the regulation at different levels of the transcription and translation of different genes allowing long-term adjustment (extensively reviewed in Saito and Posas). ${ }^{6}$ Moreover, HOG activation also produces a short-term adaptation effect, such as glycerol accumulation or the reestablishment of ionic balance. ${ }^{7-9}$

The MAPK pathways are extremely conserved among eukaryotes and are composed of a tier of three consecutive levels of activated kinases. In the HOG pathway, these levels are composed of a layer of three MAPKKKs (Ssk2, Ssk22, and Ste11) which are responsible for activating a unique MAPKK (Pbs2). After Pbs2 activation, Pbs2 phosphorylates and activates the Hog1 MAPK, ${ }^{10}$ which is a homologue of the human p38 and c-Jun N-terminal. Once phosphorylated, the Hogl is concentrated in the nucleus, where it can phosphorylate its protein targets. However, a portion of activated Hog1 is retained in the cytoplasm to regulate other cytosolic events. ${ }^{11}$

In this review, we present the latest studies on how Hog1 regulates the cell cycle progression, which is essential for cell survival preserving genomic integrity and cell viability in budding yeast.

\section{Regulation of the yeast cell cycle}

The cell cycle, whereby one initial cell divides finally into two cells, basically consists of four phases: G1 phase (for Gapl, because apparently nothing happens, but in fact cells grow in volume and decide to divide), $S$ phase (when DNA is synthesized), G2 phase (for Gap2, when cells continue growing), and $\mathrm{M}$ phase (for mitosis, when cells finally divide). After the M phase, cells again enter G1, therefore finishing a cycle.

In budding yeast, Saccharomyces cerevisiae, a highly regulated and complex network of proteins governs this process. Nevertheless, a single essential cyclin dependent kinase (CDK), Cdc28 (the functional homologue of Cdk1 in higher eukaryotes) controls cell cycle progression whose regulation is achieved mainly through the synthesis and degradation of cyclins and inhibitors, conferring its substrate specificity. ${ }^{12-14}$

At the beginning of the cell cycle, the nuclear concentration of $\mathrm{Cln} 3$ increases in relationship to the total cell mass ${ }^{15-18}$ promoting the phosphorylation of Whi5 (an ortholog of $\mathrm{Rb}$ ). This event allows the activation of the transcription factors SBF (a heterodimer of Swi4 and Swi6) and MBF (a heterodimer of
Correspondence: Xavier Escoté,

Dr Mallafré Guasch, 4 43007, Tarragona, Spain.

E-mail: xescote@gmail.com

Key words: MAPK, Hog1, CDK, cell cycle, Sic1, Swel, Hsl1, Cln1.

Acknowledgements: we apologize to colleagues for not citing all relevant papers owing to space constraints. The laboratory of JV and XE is supported by grants from the Instituto de Salud Carlos III (ISCIII) FIS11/00085 and CIBERDEM de Diabetes y Enfermedades Metabólicas Asociadas (CB07/08/0012). The laboratory of JC is supported by a grant from the former Spanish Ministry of Science and Innovation (BFU 2009-09278), now known as the Ministry of Economy and Competitiveness.

Received for publication: 16 October 2012. Revision received: 18 January 2013.

Accepted for publication: 18 January 2013.

This work is licensed under a Creative Commons Attribution NonCommercial 3.0 License (CC BYNC 3.0).

(C) Copyright J. Clotet et al., 2013

Licensee PAGEPress, Italy

MAP Kinase 2013; 2:e3

doi:10.4081/mk.2013.e

Mbpl and Swi6) inducing the transcription of a second wave of cyclins (CLN1, CLN2, CLB5, and $C L B 6$ ). The activity of $\mathrm{Cln} 1,2 / \mathrm{Cdc} 28$ stimulates bud formation however $\mathrm{Clb} 5,6 / \mathrm{Cdc} 28$ remains inhibited by the presence of Sicl. When several residues in Sicl are phosphorylated by Cln1,2/Cdc28, this leads to Sic1 recognition and ubiquitination by the SCF/Cdc4 complex; 19 and consequently targeted Sic1 destruction. When Sicl is degraded, Clb5,6/Cdc28 activity rises, phosphorylating Sicl at the same residues as those phosphorylated by $\mathrm{Cln} 1,2 / \mathrm{Cdc} 28$. Thus, Sic1 degradation accelerates due to the positive feedback loop, resulting in an abrupt rise in $\mathrm{Clb} 5,6 / \mathrm{Cdc} 28$ activity, which drives cells to $\mathrm{S}$ phase.

In $S$. cerevisiae, $\mathrm{S}$ phase is triggered fundamentally by two kinases, Clb5,6-Cdc28 and Dbf4-Cdc7, which phosphorylate specific proteins at the replication origins. DNA synthesis is instigated when $\mathrm{Clb} 5,6 / \mathrm{Cdc} 28$ phosphorylates the replication proteins Sld2 and Sld3, which are components of the pre-initiation complex. ${ }^{20,21}$ DNA synthesis begins from multiple origins that are distributed throughout the genome following a strict temporal program;22 and the assembly of the protein complexes on the replication origins is a very fine-tuned process. Initially, Cdt1, Cdc6, MCMs and proteins form the pre-replication complexes. During G1, Cdc45, Sld2, Sld3, Dpb11 and a novel replication complex GINS (Go, Ichi, Nii, and San; five, one, two, and three in Japanese), are 
loaded to constitute the pre-initiation complex. At this stage, the origins are already licensed to be activated by $\mathrm{Clb5}, 6-\mathrm{Cdc} 28$ and Dbf4-Cdc7. Those complexes phosphorylate specific target proteins on the pre-initiation complex to induce full activation of the replicative helicases and polymerases to start DNA replication from every single origin. Cell-cycle progression to the G2 phase mainly depends on another wave of cyclin production: the mitotic cyclins Clb1 and Clb2. The Mcml/SFF complex is the transcription factor that regulates expression of $C L B 1$ and $C L B 2 .{ }^{23-25}$ Entry into mitosis is driven mainly by the activity of Clb2-Cdc28, which is tightly regulated by Swel (the ortholog of higher eukaryotes Weel). Swe1 controls the Clb1,2/Cdc28 activity by direct phosphorylation at a conserved tyrosine in Cdc28, which is reactivated by the phosphatase Mih1 (the ortholog of Cdc25). Swel degradation basically depends on two independent mechanisms; the phosphorylation by $\mathrm{Clb} 2-\mathrm{Cdc} 28 ; 26,27$ and its degradation by the Hsl1, Cdc5, Elm1 and Dmal.25,28-30 When the septin ring is completed, Hsll is recruited. When bound to septins, Hsll tethers the adaptor protein Hsl7 to the bud neck, which is in turn required for Swel recruitment and is then targeted by Cdc5 leading to Swe1 destruction and release of $\mathrm{Clb2}$-Cdc28 inhibition.31,32 During vegetative growth, Swel does not appear to affect cell-cycle progression. ${ }^{33}$ However, when bud formation is impaired, Swel remains active, inactivates the $\mathrm{Clb} 1,2 / \mathrm{Cdc} 28$, and delays the cell cycle progression. ${ }^{34}$ Thus, the tight regulation of Swel phosphorylation and its subsequent degradation is critical for the cell cycle progression in G2 phase.

Exit from mitosis after chromosome segregation is controlled by a signaling cascade termed the mitotic exit network (MEN). When all chromosomes are aligned, Cdc20/anaphase promoting complex (APC) is activated; 35 promoting the degradation of Clb2 protein. ${ }^{36}$ This event represents the exit from mitosis and the start of a new cycle. MEN activation is initiated by the activation of Tem1, a G-protein. ${ }^{37}$ When cells undergo anaphase, the spindle pole body enters the daughter cell where the Ltel is located, promoting the activation of Tem1.38 Teml activates the Cdc15 kinase, a critical component of MEN, which in turn activates the Cdc14 phosphatase. Cdc14 is tightly regulated by a competitive inhibitor, Net1, which holds Cdc14 in an inactive state during most of the cell cycle except during anaphase and telophase phases. Cdc14 is released by MEN or by the fourteen early anaphase release (FEAR) network, promoting exit from mitosis. Cdc14 activates the APC/Cdh1, which ubiquitinates and degrades the remaining $\mathrm{Clb} 1$ and $\mathrm{Clb} 2$. Cdc14 also directly dephosphorylates $\mathrm{Clb} / \mathrm{Cdc28}$ substrates such as Sicl;39 which promotes Sicl stabilization and consequently $\mathrm{Clb} / \mathrm{Cdc} 28$ inhibition.

\section{Control of the G1/S transition by Hog1}

When yeast cells are exposed to high osmolarity, the Hog1 MAPK is transiently activated and a corresponding cell-cycle delay in $\mathrm{Gl}$ is produced by regulating different levels of the basic cell-cycle machinery. ${ }^{15,40-43}$ It can be demonstrated that this delay is caused by activated Hogl, and not by other effects of osmostress, by using genetic means to activate Hogl in the absence of osmostress. In contrast, when Hogl activation is sustained for a long time (by activation of the pathway using different alleles for Sln1, Pbs2 or Ssk2), cells undergo a programmed cell death that requires the action of the proteinase Nma111 and SCF/CDC4. 44

First, Hog1-mediated G1 arrest is partially mediated by down-regulation of the expression of the G1 cyclins CLN1, CLN2 and of the Scyclin CLB515 (Figure 1). The exact nature of the mechanism that represses the expression of SBF- and MBF-dependent genes under osmotic stress is still unknown. It is well described that $\mathrm{Cln} 1,2 / \mathrm{Cdc} 28$ activities are necessary to reach a threshold of Sic1 phosphorylation, with the subsequent ubiquitination by the SCF/Cdc4, and later degradation by the proteasome. Therefore, the down-regulation of CLN1 and CLN2 production might, at least in part, explain the delay in S-phase entry as a consequence of an increased accumulation of Sicl. The precise mechanism that represses the expression of the SBF- and MBF-dependent genes under osmotic stress is unclear. One possibility might be that Hogl was directly (or, by other downstream kinases, indirectly) inhibiting the activity of the transcription factors Swi4/Swi6 by phosphorylation. Another possibility could be that Hogl directly phosphorylates Whi5, the ortholog of mammalian retinoblastoma $(\mathrm{Rb})$. In any case, the exact mechanism by which Hog1 is able to repress G1 cyclin expression remains to be explored.

Second, Hogl arrests cells in G1 by an alternative mechanism: the direct phosphorylation of Sic1.40 When Hog1 is activated, it interacts with Sicl and phosphorylates the CDK inhibitor into a single residue (T173) at the carboxyl-terminal region of Sic1. This phosphorylation somehow interferes with the binding of Sicl with the SCF/Cdc4, inhibiting Sic1 ubiquitination and consequently its degrada tion ${ }^{40,45,46}$ (Figure 1). Thus, Sic1 is stabilized, which implies an inhibition of $\mathrm{Clb} 5,6 / \mathrm{Cdc} 28$ delaying the G1/S transition. The stabilization of Sicl and the consequent Hog1-dependent G1 arrest is essential for the adaptive response to osmostress since cells lacking Sicl or carrying a non-phosphorylatable allele of Sic1 (T173A) display reduced viability in high osmolarity as a result of genomic instability ${ }^{40}$ Therefore, the complex and strict Hogl control over the G1/S network clearly illustrates the need for cell adaptation to osmostress prior to progressing to $\mathrm{S}$ phase. Mathematical modelling supported by quantitative in vivo experiments allowed defining and quantifying the temporal role and the direct contribution of the individual components of the G1/S transition

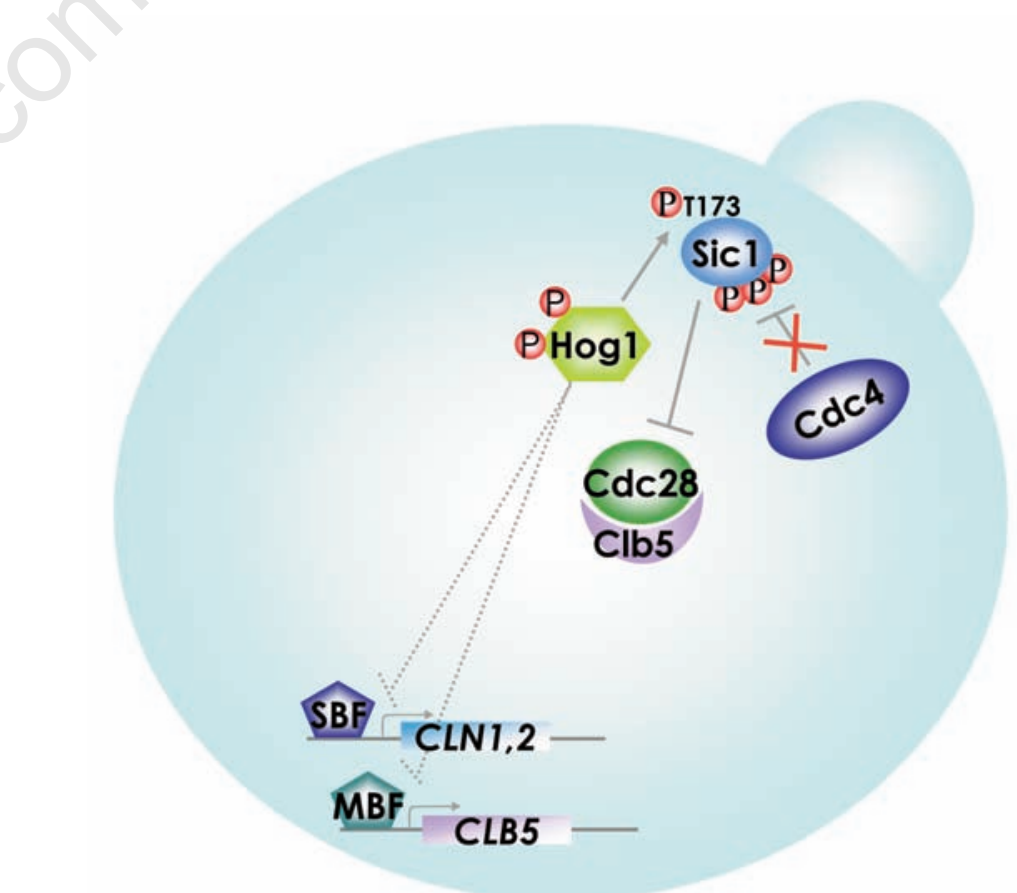

Figure 1. Schematic diagram of the control of the cell cycle progression by Hogl at the different phases of the cell cycle. 
controlled by Hog1.43 Hog1-induced inhibition of the transcription of the gene encoding cyclin Clb5, rather than that of the gene encoding $C$ ln2, prevented entry into $S$ phase upon osmostress. By controlling the accumulation of specific cyclins, Hog1 delayed bud morphogenesis (through Clns) and delayed DNA replication (through Clb5). Hog1-mediated phosphorylation and degradation of Sicl at Start prevented residual activity of the cyclin/CDK complex Clb5/Cdc28 from initiating DNA replication before adaptation to the stress. 43

The triple targeting of $C L N 1 / 2, C L B 5$ and Sic1 ensures a G1 transient arrest at any stage of G1 in response to osmostress. In this way, Hog1 inhibits cyclin expression in cells in early G1 phase, and later in G1, Hogl inhibits Sicl degradation. Of the three cyclin genes whose transcription is inhibited by Hogl, inhibition of CLN1,2 expression delays bud morphogenesis and spindle pole body duplication, and inhibition of CLB5 expression delays DNA replication and consequently entry into $\mathrm{S}$ phase. Later in G1, when these cyclins are already expressed, inhibition of cyclin expression can no longer prevent cell-cycle progression. Instead, Hog1-mediated phosphorylation and inhibition of the degradation of Sic1 prevents active Clb5/Cdc28 from initiating DNA replication. Therefore, Hog1 activation managed to control the basic processes that occur in G1 allowing replication only when cells were adapted to the new environmental conditions. Thus, these distinct mechanisms that operate at different time points ensure that no premature entry into $S$ phase occurs under osmostress conditions. With regard to G1 entry, a role of Hogl modulating re-entry into cell cycle from a resting state situation as $\mathbf{G} 0$ phase; 47 it has recently been described. Hogl deficient cells show a delay in entering the mitotic cell cycle from the stationary phase. Moreover, when cells enter the mitotic cell cycle after being in the stationary phase, Hog1 is rapidly activated and concentrates in the nucleus where it modifies the expression of several genes. After discarding that these effects were not caused by an osmotic process, what the stimulus is that activates Hogl at the entrance to cell cycle remains to be elucidated. Hogl activation in this process may be by sensing the presence of glucose, nitrogen or phosphate. On the other hand, what are the targets of Hog1 to facilitate re-entry cycle? One possible target would be Rim15, which is a key element in establishing G0 phase. Nevertheless, further experiments are needed to prove this hypothesis.

\section{Regulation of the S phase by} high osmolarity glycerol 1

The Hog1 SAPK is not only important for regulating the G1/S transition but it also plays a crucial role once the cells are already in Sphase in delaying DNA replication in response to osmostress. ${ }^{48}$ During $\mathrm{S}$ phase, the genome replicates, which is a highly ordered process involving many proteins. Cells have evolved a specific S-phase checkpoint to cope with multiple genotoxic agents that endanger the proper progression and completion of DNA replication. The S-phase checkpoint is mediated by Rad53 which safeguards DNA replication and preserves genomic integrity. In the presence of DNA damage or replication stresses, the Rad53-dependent checkpoint pathway delays $\mathrm{S}$ phase avoiding late origin firing. ${ }^{49-52}$ Strikingly, Hogl-dependent arrest in S phase upon osmostress is independent of the known Rad53-dependent checkpoint pathway, suggesting that there must be a novel S-phase checkpoint pathway that delays DNA replication in the absence of DNA damage or replication stress. ${ }^{48}$

When cells are stressed in early $\mathrm{S}$ phase, Hogl controls the $S$ phase progression by delaying the expression of the S-phase cyclins CLB5. If cells are stressed later in $\mathrm{S}$ phase, Hogl physically interacts with several components of the replication complex, as well as delaying phosphorylation of the Dpb2 subunit of the DNA polymerase. ${ }^{48}$ Although the molecular mechanism by which Hog1 delays DNA replication remains unclear, this function is clearly independent of the SAPK cell cycle direct targets Sicl and Hsll and the S-phase DNA Rad53 checkpoint.

In response to osmostress, Hogl orchestrates a fast and transient activation of transcription of hundreds of stress-responsive genes essential for adaptation to stress. ${ }^{2}$ Adaptive responses to osmostress require the induction of the expression of a very large number of genes. It is therefore conceivable that initiating or ongoing replication might occur on the genes that are being transcribed for adaptation. It is easy to see that if the large replication complex and the transcription complex attempted to occupy the same space, they would interfere with each other's function. In fact, it has been shown that collision between RNA Pol II and DNA polymerase induces transcription-associated recombination. ${ }^{53-55}$ Thus, delaying replication in response to osmostress must be important both to provide proper adaptive gene expression and to prevent genomic instability.

\section{Regulation of $\mathbf{G} 2$ phase by high osmolarity glycerol 1}

Activation of Hogl induces a transient cellcycle delay in G2 progression by the decrease in $\mathrm{Clb} 2 / \mathrm{Cdc} 28$ activity as a consequence of indirect Swe1 accumulation and the down-regulation of CLB2 transcription ${ }^{56,57}$ (Figure 2). The mechanism required to decrease Clb2/Cdc28 activity is well understood: Hog1 acts on the machinery of the morphogenetic checkpoint that controls the CDK inhibitor levels of Swel. Activated Hogl interacts and directly phosphorylates Hsl1 in a single residue (S1220) within the Hsl7-docking site, which promotes the delocalization of Hsl7 from the bud neck that results in Swel accumula-

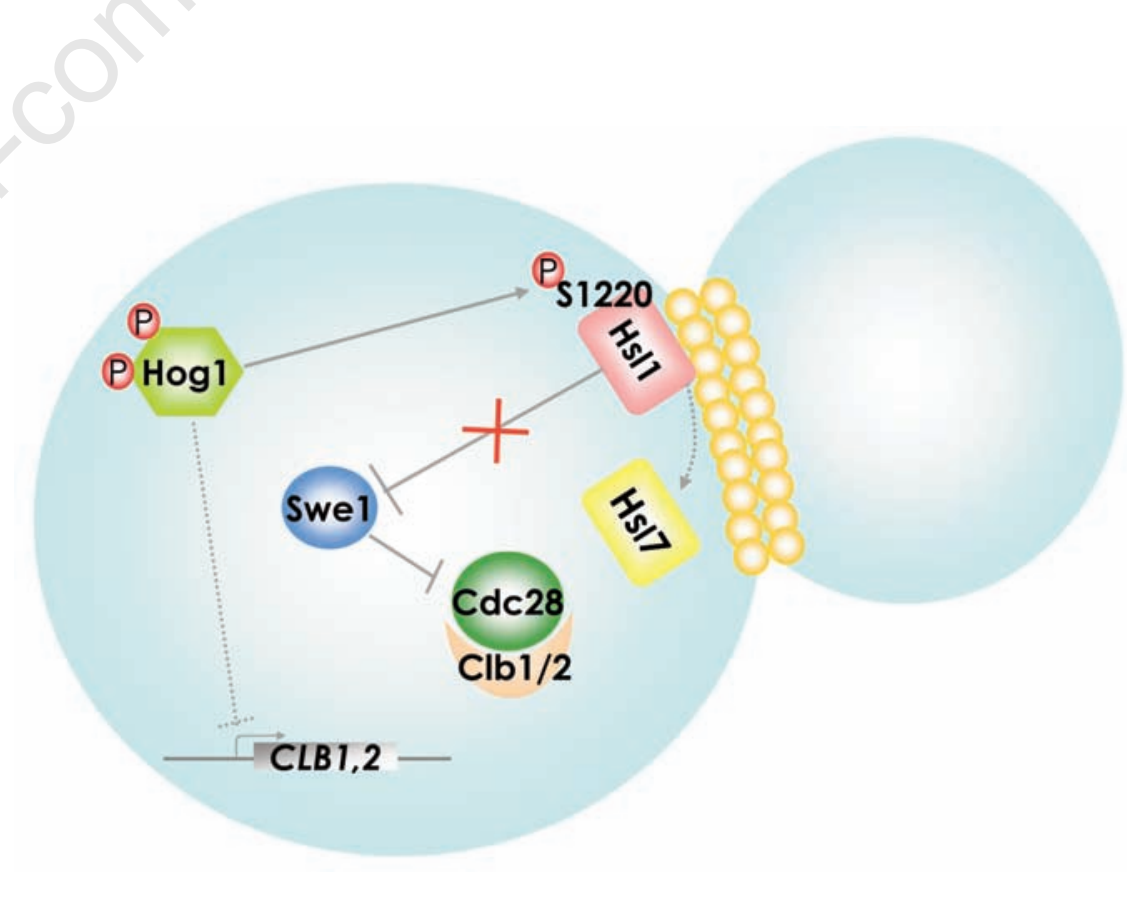

Figure 2. Schematic diagram of the control of the cell cycle progression by Hog1 at the G2 phase. 
tion. ${ }^{57}$ Upon Hog 1 activation, cells containing a non-phosphorylatable allele of Hsl1 (S1220A) are unable to promote Hsl7 delocalization. These mutants cannot accumulate enough Swe1 inhibitor and fail to arrest in G2 and render cells osmosensitive as a consequence of premature progress into $M$ phase. Interestingly, whereas the morphogenetic checkpoint monitors the absence of a septin ring, which implies the delocalization of Hsl1, Hsl7, and Swel at the bud neck, Hogl activation specifically delocalizes Hsl7, and consequently Swe1, without affecting the Hsll or the subcellular localization of septins. ${ }^{57}$ Thus, the tight regulation of Swe1 localization, phosphorylation and its subsequent degradation are critical for the timely activation of the Clb2/Cdc28 complex and ensure the proper assembly of the septin ring throughout G2 progression. In contrast, the mechanisms that Hogl employs to down-regulate CLB2 transcription levels are not known, but this could be a secondary effect based on the decrease in Cdc28/Clb2 activity. Furthermore, the combined absence of the CDK inhibitors SIC1 and SWE1 results in a synergistic phenotype of osmosensitivity; 57 highlighting the relevance of the cell cycle control by Hogl at different stages of the cell cycle. In any case, Hog1 activation affects not only the activity of Cdc28/Clb2 but also the Cln2 levels by a downregulation of CLB2 transcription ${ }^{56,57}$ (Figure
2). The mechanism that leads to the repression of CLB2 is unknown, but it is tempting to speculate that the transcriptional activators and Fkh1 Fkh2 will be involved. Moreover, it is necessary to study the possible effect of Hog 1 on the Cdc28 complexes with the cyclins Clb3 Clb4.

\section{Exit from mitosis}

Exit from mitosis could also be regulated by the Hog1 MAPK under osmotic stress. In response to osmostress, MEN mutants exit from mitosis in a manner that is dependent on Hog1. In such MEN mutants, the HOG pathway seems to regulate exit from mitosis by promoting the function of the FEAR network that activates Cdc14, although the exact mechanism remains unclear ${ }^{58}$ (Figure 3). What is well demonstrated, in a situation of osmotic stress, is that $\mathrm{M}$ phase synchronized cells circulate more rapidly during anaphase to telophase transition (our unpublished observations, 2005). This suggests that Hogl activation at this point of the cell cycle could be sped up by M. (which is contrary to the observed transitions of G1, S and G2). Nevertheless, it will be necessary to perform further experiments to find the possible target/s of Hogl at this point. Another important area to explore the role of
Hog1 is what happens at the end of the cell cycle, at cytokinesis, which is a point in the cell cycle when cells are especially sensitive to osmotic stress. In our opinion, after an osmotic shock, Hog1 must avoid premature entry into cytokinesis. Nevertheless, again further experiments are needed to validate these speculations.

\section{Conclusions}

The evidence presented in this review presents Hogl as a guardian to protect the cell from external stresses at any stage of the cycle in which it is found. Indeed, several well-known protein targets of Hogl covering the entire cell cycle (Figure 3). Clearly the mitosis phase remains to be explored, but it is tempting to speculate that Hogl must be regulating some processes at such a critical moment for cell viability.

In any case, in the cell cycle phases in which Hogl acts as a main regulator, the MAP kinase blocks the cell cycle progression with a delay of 20-30 minutes, which is the time required to activate various metabolic enzymes, such as Pfk2;59 Gpd160 or Gph1;61 which leads to an accumulation of glycerol in the cell.

Nevertheless, this evidence gives rise to other important questions:

After cells are acclimatized to the new environmental conditions and have therefore increased the inner levels of glycerol, which are the mechanisms that can revoke the Hogl delay? In other words, what are the different protein phosphatases that dephosphorylate the Hogl targets, and consequently allow cells to continue in the cell cycle progression?

The idea that osmotic stress causes a cycle arrest to facilitate adaptation is transportable to other stresses: desiccation, oxidative, heat, etc. In $S$. cerevisiae, it seems that Hogl only responds to osmotic stress, but in $S$. pombe and in mammalian cells the Hogl orthologs are activated by oxidative stress also indicating that different stimuli lead to the same protective effects.

Are these mechanisms evolutionarily preserved? It is legitimate to speculate that these processes are indispensable, and consequently, different organisms, from yeast to human, must respond in a similar manner to these injuries.

In fact, it has been reported that the mammal-Hog1-ortholhog p38 phosphorylates the CDK inhibitor p2162 and down-regulates the cyclin D1 expression ${ }^{63}$ which implies a clear parallelism with what is happening in yeast.

In which situations may be relevant this mechanism? i) yeast cells live on grape skin, consequently yeast cells are submitted to continuously changing conditions of dedication

Figure 3. Schematic diagram of the control of the cell cycle progression by Hogl at the different phases of the cell cycle. 
and humidity gain; ii) another situation is when the physiological grape breaks, releasing the juice which implies that yeast cells suffer an osmotic shock.

Are these mechanisms of osmoadaptation in mammals really relevant? Regarding mammals, their cells are constantly subjected to osmotic changes. Perhaps the most obvious occurs in the lung epithelium cells (which can be found in the sudden humidity changes) or cells from the lining of the bladder where it has been shown that p38 is activated and stops the G2 cycle. In addition, in a very recent study, osmotic stress has been reported to promote the phosphorylation of the CDK inhibitor p57 by 38 , facilitating the inhibitor accumulation and, consequently, delaying the G1 transition. 64

In summary, we can conclude that proper adaptation to osmostress seems to be a prerequisite for advancing to the following cell cycle phase, and thus, it seems rational to think that the same MAPK, Hogl, is able to control the progression for the different cell cycle phases. This regulation might be critical to certify that cells at any stage of the cell cycle are competent to progress to the next phase with the appropriate adaptive responses.

\section{References}

1. de Nadal E, Posas F. Multilayered control of gene expression by stress-activated protein kinases. EMBO J 2010;29:4-13.

2. de Nadal E, Ammerer G, Posas F. Controlling gene expression in response to stress. Nat Rev Genet 2011;12:833-45.

3. de Nadal E, Alepuz PM, Posas F. Dealing with osmostress through MAP kinase activation. EMBO Rep 2002;3:735-40.

4. Hohmann S. Osmotic stress signaling and osmoadaptation in yeasts. Microbiol Mol Biol Rev 2002;66:300-72.

5. Yancey P, Clark M, Hand S, et al. Living with water stress: evolution of osmolyte systems. Science 1982;217:1214-22.

6. Saito H, Posas F. Response to hyperosmotic stress. Genetics 2012;192:289-318.

7. Albertyn J, Hohmann S, Thevelein JM, Prior BA. GPD1, which encodes glycerol-3phosphate dehydrogenase, is essential for growth under osmotic stress in Saccharomyces cerevisiae, and its expression is regulated by the high-osmolarity glycerol response pathway. Mol Cell Biol 1994;14:4135-44.

8. Tamas MJ, Luyten K, Sutherland FC, et al. Fps1p controls the accumulation and release of the compatible solute glycerol in yeast osmoregulation. Mol Microbiol 1999:31:1087-104.

9. Proft M, Struhl K. MAP kinase-mediated stress relief that precedes and regulates the timing of transcriptional induction. Cell 2004;118:351-61.

10. Brewster JL, de Valoir T, Dwyer ND, et al. An osmosensing signal transduction pathway in yeast. Science 1993;259:1760-3.

11. Reiser V, Ruis H, Ammerer G. Kinase activity-dependent nuclear export opposes stress-induced nuclear accumulation and retention of Hog1 mitogen-activated protein kinase in the budding yeast Saccharomyces cerevisiae. Mol Biol Cell 1999;10:1147-61.

12. Hartwell LH, Mortimer RK, Culotti J, Culotti M. Genetic control of the cell division cycle in yeast: V. Genetic analysis of CDC mutants. Genetics 1973;74:267-86.

13. Rosenthal ET, Hunt T, Ruderman JV. Selective translation of mRNA controls the pattern of protein synthesis during early development of the surf clam, Spisula solidissima. Cell 1980;20:487-94.

14. Nasmyth K. Control of the yeast cell cycle by the Cdc28 protein kinase. Curr Opin Cell Biol 1993;5:166-79.

15. Belli G, Gari E, Aldea M, Herrero E. Osmotic stress causes a G1 cell cycle delay and downregulation of Cln3/Cdc28 activity in Saccharomyces cerevisiae. Mol Microbiol 2001;39:1022-35.

16. Gari E, Volpe T, Wang $\mathrm{H}$, et al. Whi3 binds the mRNA of the G1 cyclin CLN3 to modulate cell fate in budding yeast. Genes Dev 2001;15:2803-8.

17. Chen KC, Csikasz-Nagy A, Gyorffy B, et al. Kinetic analysis of a molecular model of the budding yeast cell cycle. Mol Biol Cell 2000;11:369-91.

18. Vergés E, Colomina N, Garí E, et al. Cyclin $\mathrm{Cln} 3$ is retained at the ER and released by the $\mathrm{J}$ chaperone Ydj1 in late $\mathrm{G} 1$ to trigger cell cycle entry. Mol Cell. 2007;26:649-62.

19. Verma R, Annan RS, Huddleston MJ, et al. Phosphorylation of Siclp by G1 cdk required for its degradation and entry into S phase. Science 1997;278:455-60.

20. Masumoto H, Muramatsu S, Kamimura Y, Araki H. S-Cdk-dependent phosphorylation of Sld2 essential for chromosomal DNA replication in budding yeast. Nature 2002;415:651-5.

21. Tanaka S, Umemori T, Hirai K, et al. CDKdependent phosphorylation of SId2 and Sld3 initiates DNA replication in budding yeast. Nature 2007;445:328-32.

22. Raghuraman MK, Brewer BJ, Fangman WL. Cell cycle dependent establishment of a late replication program. Science 1997;276:806-9.

23. Althoefer H, Schleiffer A, Wassmann K, et al. $\mathrm{Mcml}$ is required to coordinate G2-specific transcription in Saccharomyces cerevisiae. Mol Cell Biol 1995;15:5917-28.

24. Maher M, Cong F, Kindelberger D, et al.
Cell cycle regulated transcription of the CLB2 gene is dependent on Mcml and a ternary complex factor. Mol Cell Biol 1995;15:3129-37.

25. Jorgensen P, Tyers M. The fork'ed path to mitosis. Genome Biol 2000;1: REVIEWS 1022.

26. Asano S, Park JE, Sakchaisri K, et al. Concerted mechanism of Swe1/Weel regulation by multiple kinases in budding yeast. EMBO J 2005;24:2194-204.

27. Harvey SL, Charlet A, Haas W, et al. Cdk1dependent regulation of the mitotic inhibitor Weel. Cell 2005;122:407-20.

28. Hanrahan J, Snyder M. Cytoskeletal activation of a checkpoint kinase. Mol Cell 2003;12:663-73.

29. Merlini L, Fraschini R, Boettcher B, et al. Budding yeast dma proteins control septin dynamics and the spindle position checkpoint by promoting the recruitment of the Elm1 kinase to the bud neck. PLoS Genet 2012;8:e1002670.

30. Raspelli E, Cassani C, Lucchini G, Fraschini R. Budding yeast Dmal and Dma2 participate in regulation of Swe1 levels and localization. Mol Biol Cell 2011;22:2185-97.

31. Versele M, Thorner J. Some assembly required: yeast septins provide the instruction manual. Trends Cell Biol 2005;15:414-24.

32. Lew DJ. The morphogenesis checkpoint: How yeast cells watch their figures. Curr Opin Cell Biol 2003;15:648-53.

33. Amon A, Surana U, Muroff I, Nasmyth K. Regulation of p34CDC28 tyrosine phosphorylation is not required for entry into mitosis in S. cerevisiae. Nature 1992;355: 368-71.

34. McMillan JN, Sia RA, Bardes ES, Lew DJ. Phosphorylation independent inhibition of Cdc28p by the tyrosine kinase Swelp in the morphogenesis checkpoint. Mol Cell Biol 1999;19:5981-90.

35. Hwang LH, Lau LF, Smith DL, et al. Budding yeast Cdc20: A target of the spindle checkpoint. Science 1998;279:1041-4.

36. Baumer M, Braus GH, Irniger S. Two different modes of cyclin clb2 proteolysis during mitosis in Saccharomyces cerevisiae. FEBS Lett 2000;468:142-8.

37. Jaspersen SL, Charles JF, Morgan DO. Inhibitory phosphorylation of the APC regulator Hctl is controlled by the kinase Cdc28 and the phosphatase Cdc14. Curr Biol 1999;9:227-36.

38. Pereira G, Hofken T, Grindlay J, et al. The Bub2p spindle checkpoint links nuclear migration with mitotic exit. Mol Cell 2000;6:1-10.

39. Stegmeier F, Amon A. Closing mitosis: The functions of the Cdc14 phosphatase and its regulation. Annu Rev Genet 2004;38:203-32. 
40. Escote X, Zapater M, Clotet J, Posas F. Hog1 mediates cell-cycle arrest in G1 phase by the dual targeting of Sicl. Nat Cell Biol 2004;6:997-1002.

41. Clotet J, Posas F. Control of cell cycle in response to osmostress lessons from yeast. Methods Enzymol 2007;428:63-76.

42. Zapater M, Sohrmann M, Peter M, et al. Selective requirement for SAGA in Hog1mediated gene expression depending on the severity of the external osmostress conditions. Mol Cell Biol 2007;27:3900-10.

43. Adrover M, Zi Z, Duch A, et al. Timedependent quantitative multicomponent control of the G1-S network by the stressactivated protein kinase Hogl upon osmostress. Sci Signal 2011;4:ra63.

44. Vendrell A, Martínez-Pastor M, GonzálezNovo A, et al. Sir2 histone deacetylase prevents programmed cell death caused by sustained activation of the Hogl stressactivated protein kinase. EMBO Rep 2011; 12:1062-8.

45. Zapater M, Clotet J, Escoté X, Posas F. Control of cell cycle progression by the stress-activated Hog1 MAPK. Cell Cycle 2005;4:6-7.

46. Duch A, de Nadal E, Posas F. The p38 and Hog1 SAPKs control cell cycle progression in response to environmental stresses. FEBS Lett 2012;586:2925-31.

47. Escote X, Miranda M, Rodríguez-Porrata B, et al. The stress-activated protein kinase Hog1 develops a critical role after resting state. Mol Microbiol 2011;802:423-35.

48. Yaakov G, Duch A, García-Rubio M, et al. The stress-activated protein kinase Hogl mediates $\mathrm{S}$ phase delay in response to osmostress. Mol Biol Cell 2009;20:3572-82.
49. Dahmann C, Diffley JF, Nasmyth KA. Sphase-promoting cyclindependent kinases prevent re-replication by inhibiting the transition of replication origins to a prereplicative state. Curr Biol 1995;5:1257-69.

50. Lopez-Mosqueda J, Maas NL, Jonsson ZO, et al. Damage-induced phosphorylation of Sld3 is important to block late origin firing. Nature 2010;467:479-83.

51. Zegerman P, Diffley JF. Checkpointdependent inhibition of DNA replication initiation by SId3 and Dbf4 phosphorylation. Nature 2010;467:474-8.

52. Duch A, Palou G, Jonsson Z0, et al. A Dbf4 mutant contributes to bypassing the Rad53- mediated block of origins of replication in response to genotoxic stress. $\mathrm{J}$ Biol Chem 2011;286:2486-91.

53. Aguilera A. The connection between transcription and genomic instability. EMBO J 2002;21:195-201.

54. Prado F, Aguilera A. Impairment of replication fork progression mediates RNA polII transcription-associated recombination. EMBO J 2005;24:1267-76.

55. Aguilera A, Gómez-González B. Genome instability: a mechanistic view of its causes and consequences. Nat Rev Genet 2008;9:204-17.

56. Alexander MR, Tyers M, Perret M, et al. Regulation of cell cycle progression by Swelp and Hoglp following hypertonic stress. Mol Biol Cell 2001;12:53-62.

57. Clotet J, Escote X, Adrover MA, et al. Phosphorylation of Hsll by Hogl leads to a G2 arrest essential for cell survival at high osmolarity. EMBO J 2006;25:2338-46.

58. Reiser V, D’Aquino KE, Ee LS, Amon A. The stress-activated mitogen activated protein kinase signaling cascade promotes exit from mitosis. Mol Biol Cell 2006;17:313646.

59. Dihazi H, Kessler R, Eschrich K. High osmolarity glycerol (HOG) pathwayinduced phosphorylation and activation of 6-phosphofructo-2-kinase are essential for glycerol accumulation and yeast cell proliferation under hyperosmotic stress. J Biol Chem 2004;279:23961-8.

60. Albertyn J, Hohmann S, Thevelein JM, Prior BA. GPD1, which encodes glycerol-3phosphate dehydrogenase, is essential for growth under osmotic stress in Saccharomyces cerevisiae, and its expression is regulated by the high-osmolarity glycerol response pathway. Mol Cell Biol 1994;14:4135-44.

61. Sunnarborg SW, Miller SP, Unnikrishnan I, LaPorte DC. Expression of the yeast glycogen phosphorylase gene is regulated by stress-response elements and by the HOG MAP kinase pathway. Yeast 2001;18:150514.

62. Todd DE, Densham RM, Molton SA, et al. ERK1/2 and p38 cooperate to induce a p21CIP1-dependent G1 cell cycle arrest. Oncogene 2004;23:3284-95.

63. Lavoie JN, L'Allemain G, Brunet A, et al. Cyclin D1 expression is regulated positively by the p42/p44MAPK and negatively by the p38/HOGMAPK pathway. J Biol Chem 1996;271:20608-16.

64. Joaquin M, Gubern A, González-Nuñez D, et al. The p57 CDKi integrates stress signals into cell-cycle progression to promote cell survival upon stress. EMBO J 2012;31:2952-64. 